\title{
Ligustrazine Injection for Chronic Pulmonary Heart Disease: A Systematic Review and Meta-Analysis
}

\author{
Li Jian-sheng, ${ }^{1,2}$ Wang Hai-feng, ${ }^{1}$ Bai Yun-ping, ${ }^{1}$ Li Su-yun, ${ }^{1}$ Yu Xue-qing, ${ }^{1}$ and Li Ya ${ }^{1}$ \\ ${ }^{1}$ Department of Respiratory Medicine, The First Affiliated Hospital of Henan University of Traditional Chinese Medicine, Zhengzhou \\ 450000, China \\ ${ }^{2}$ The Geriatric Department of Henan University of Traditional Chinese Medicine, Zhengzhou 450008, China
}

Correspondence should be addressed to Li Jian-sheng, li_js8@163.com

Received 25 February 2011; Accepted 10 May 2011

Academic Editor: Virginia S. Martino

Copyright (C) 2012 Li Jian-sheng et al. This is an open access article distributed under the Creative Commons Attribution License, which permits unrestricted use, distribution, and reproduction in any medium, provided the original work is properly cited.

\begin{abstract}
Objective. This study was intended to evaluate the efficacy and safety of ligustrazine injection for chronic pulmonary heart disease (CPHD). Method. Randomized controlled trials (RCTs) of clinical therapeutic studies on CPHD when using ligustrazine injection were included. Searches were applied to the following electronic databases: the PubMed, the Cochrane Library, EMBASE, $\mathrm{CBM}$, and AMED. No language restriction was used. All trials included were analyzed according to the criteria of the Cochrane Handbook. Review Manager 5.0 software was used for data analysis. Result. 34 RCTs with low methodological quality were included. Compared to conventional medicine treatment alone, ligustrazine injection plus conventional medicine treatment showed improvement in New York Heart Association classification of clinical status (Odds ratio 0.22; 95\% CI 0.17 to 0.28) and depression of pulmonary artery hypertension (weighted mean difference $-4.77 ; 95 \% \mathrm{CI}-5.85$ to -3.68 ). Three studies had reported adverse events. No serious adverse effects were reported. Conclusion. While there is some evidence that suggests potential effectiveness of ligustrazine injection for CPHD, the results were limited by the methodological flaws of the studies. High quality studies are needed to provide clear evidence for the future use of ligustrazine injection.
\end{abstract}

\section{Introduction}

Chronic pulmonary heart disease (CPHD) is a rising major public health problem worldwide [1]. In China, many traditional Chinese patent medicines (TCPMs) are regularly used in CPHD patients in hospital. Few studies reporting the effectiveness and safety of many commonly used TCPMs for cor pulmonale have been published in English. Sichuan lovage rhizome is one kind of traditional Chinese herbal medicines, which has the function of promoting blood circulation and qi flowing, dispelling pathogenic wind and relieving pain. It has long been used for the treatment of heart disease and cerebral disease in China. Ligustrazine is an isolated alkali extracted from Sichuan lovage rhizome. It is the formula is 2,3,5,6-tetramethyl pyrazine. It has effect of dilating blood vessels, inhibiting platelet aggregation and preventing thrombopoiesis. There are two common types: ligustrazine phosphate and ligustrazine hydrochloride. Several clinical trials have shown that ligustrazine injection might have the therapeutic effect of improving cardiac function in patients with CPHD. However, the quality of these trials has not been assessed systematically. Furthermore, the spontaneous evolution of CPHD is difficult to predict. A systematic paper will be beneficial for current practice and directive for continuing research for new treatment regimens. The objective of this review was to assess both positive and negative effects of ligustrazine injection plus conventional medicine treatment versus conventional medicine treatment in all types of CPHD in adults.

\section{Materials and Methods}

2.1. Eligibility Criteria. The eligibility criteria for conducting the survey included administration of ligustrazine hydrochloride injection for the treatment of CPHD and duration of treatment and also included trials involving patients of any age, sex, or CPHD stage. However, we excluded 
pharmacokinetic studies, nonrandomized evaluations, and animal/laboratory studies.

2.2. Search Strategy. Two reviewers (L. S. Y. and W. Z. W.) identified relevant randomised controlled trials independently, including all languages, by a systematic search of PubMed (1990-2010), Chinese Biomedical Database (CDROM, 1990-2010), EMBASE (1990 to December 2010), AMED (1990 to December 2010), Chinese BioMedical Literature Database (CBM, 1990 to December 2010), the Cochrane Library (Issue 2, 2010), and BIOSIS (1997 to 2008). With the following terms: (cor pulmonale or chronic pulmonary heart disease) AND (Ligustrazine hydrochloride injection or ligustrazine hydrochloride injection). We used the wild card term " $*$ " to enhance the sensitivity of our search strategy. Reviewers scanned the bibliographies of all retrieved trials and other relevant publications, including reviews and meta-analyses, to ensure a thorough search.

2.3. Study Selection and Data Extraction. Two reviewers (L. S. Y. and L. Y.) extracted data and evaluated data's quality and content independently. We conducted data extraction using a standardized procedure. Initially, abstracts were screened to exclude obviously ineligible reports, and then all remaining articles were reviewed. We classified trials and abstracts according to drug, patient characteristics, study design, and therapy duration. Reviewing study design included the following criteria: methods of sequence generation, allocation concealment, complete description of those who were blinded [2], and use of intention-to-treat analysis and whether the trial was stopped prior to the planned duration, all methodological features in addition capable of impacting effect sizes.

The primary outcome measures included (1) New York Heart Association classification (NYHA) of clinical status; (2) adverse events. The secondary outcome measures included (1) death, (2) hemodynamics, (3) quality of life as measured by various instruments.

The data was entered into an electronic database by the two reviewers separately, avoiding duplicate entries; in the case where the two entries did not match, an inspection will be conducted, and a third person may be involved for verification. In order to obtain full information regarding conference abstracts, we had contacted the study authors by email and/or telephone communication.

2.4. Data Analysis. The statistical package (RevMan 5.0) provided by the Cochrane Collaboration was used to analyze the data. Dichotomous data was presented as Odds Ratio (OR), with $95 \%$ confidence intervals (CIs). Continuous outcomes were presented as weighted mean difference, with 95\% CI. Analyses were performed by intention-to-treat where possible. Otherwise, for dichotomous outcomes, patients with incomplete or missing data were included in sensitivity analyses by counting them alternately as treatment failures or successes. For continuous variables we used simple sensitivity analysis to test how robust the results were under different assumptions about what might have happened to patients with missing data.

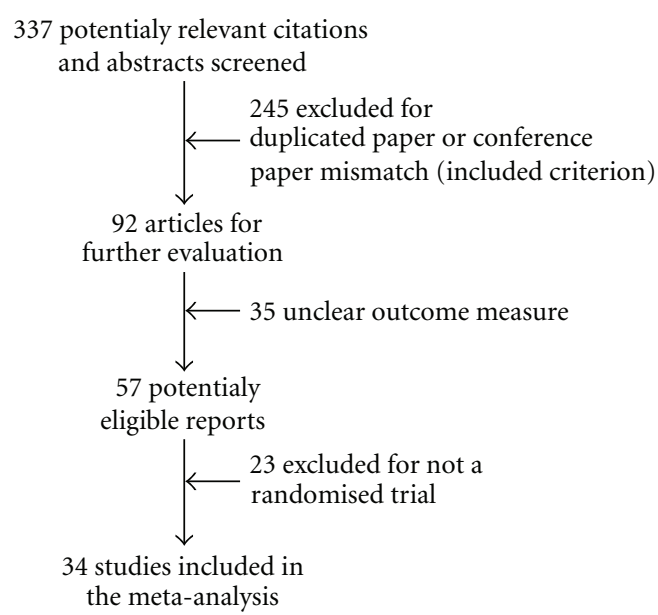

Figure 1: The Quality of Reporting of Meta-analyses flow diagram for the meta-analysis.

Meta-analysis was only performed within comparisons where individual trials were compared with similar treatment and control interventions.

\section{Results}

3.1. Study Identification and Characteristics. An initial search identified 337 potentially relevant articles. A total of 245 articles were initially excluded because of duplicate publication, and then 35 articles were excluded because they did not meet our inclusion criteria. From the identified 57 potentially eligible reports, 23 articles out of 57 were excluded for further assessment. The most frequent reasons for exclusion were other types of intervention examined, either no clinical outcomes reported, or not a randomized trial so that a total of 34 studies (2319 participants) were included into the review. All studies were published in Chinese. Figure 1 summarized the search results based on the quality of reporting of meta-analyses flow diagram. The bibliographic details of these trials are given in Table 1.

The age of patients in the included studies ranged from 37 to 85 years old. Most trials included more males (54\% to $89 \%$ ) than females; only 1 trial included more females $(55 \%)$. All included trials applied standard western medicine diagnostic criteria for CPHD, although some trials also used TCM criteria in addition. The dose range of ligustrazine hydrochloride injection was from $60 \mathrm{mg}$ to $800 \mathrm{mg}$ and dose route was intravenous injection in all included studies. The total duration of treatment varied from 7 to 15 days.

None of the trials have undergone assessment of patients' routine living ability after treatment; some trials had measurement of blood gas analysis and hemodynamic parameters.

Only ligustrazine injection was studied in these included trials. None of the trials was randomized, double-blind, placebo controlled. All included trials were designed to compare ligustrazine hydrochloride injection plus conventional treatment with the conventional treatment alone. 


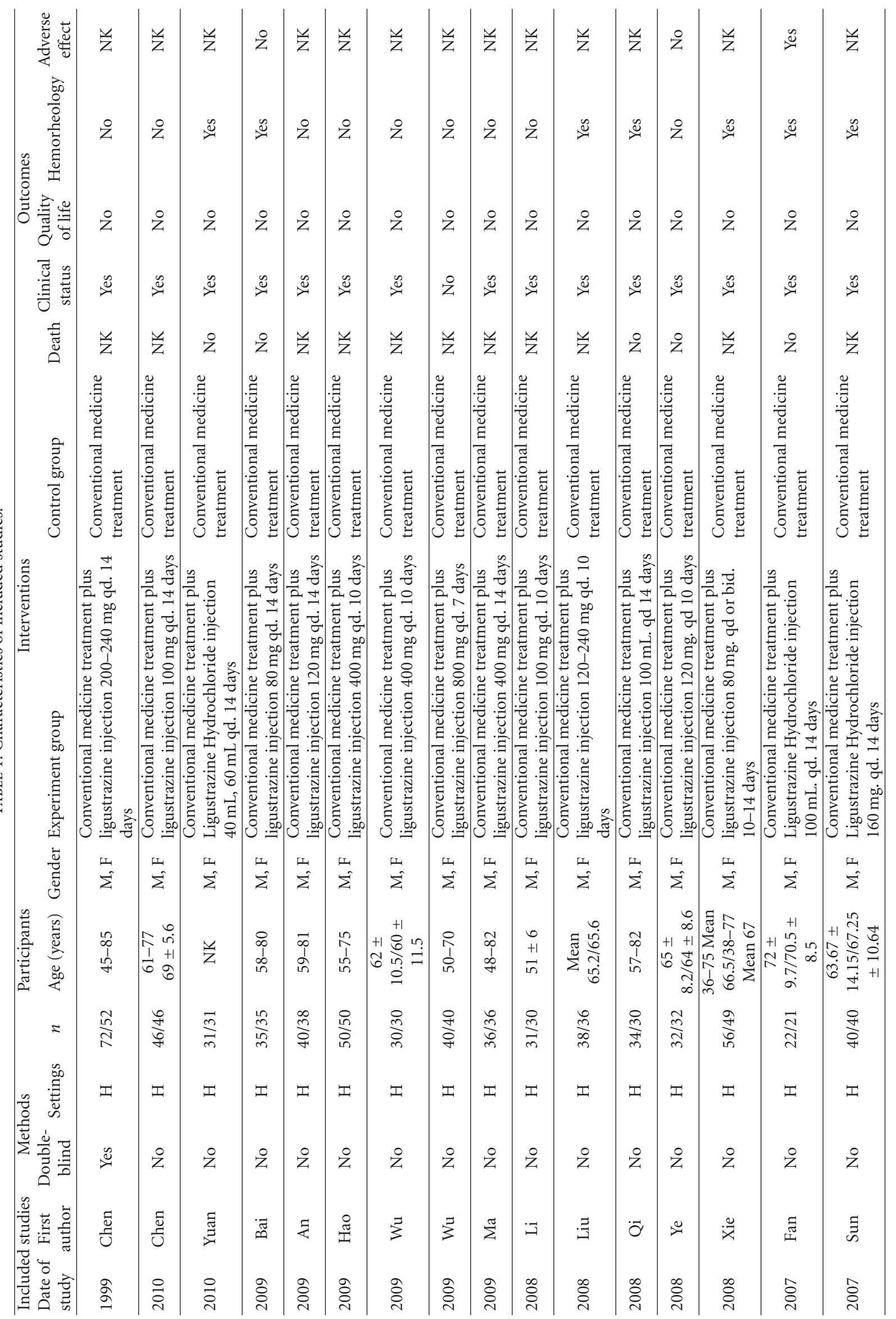




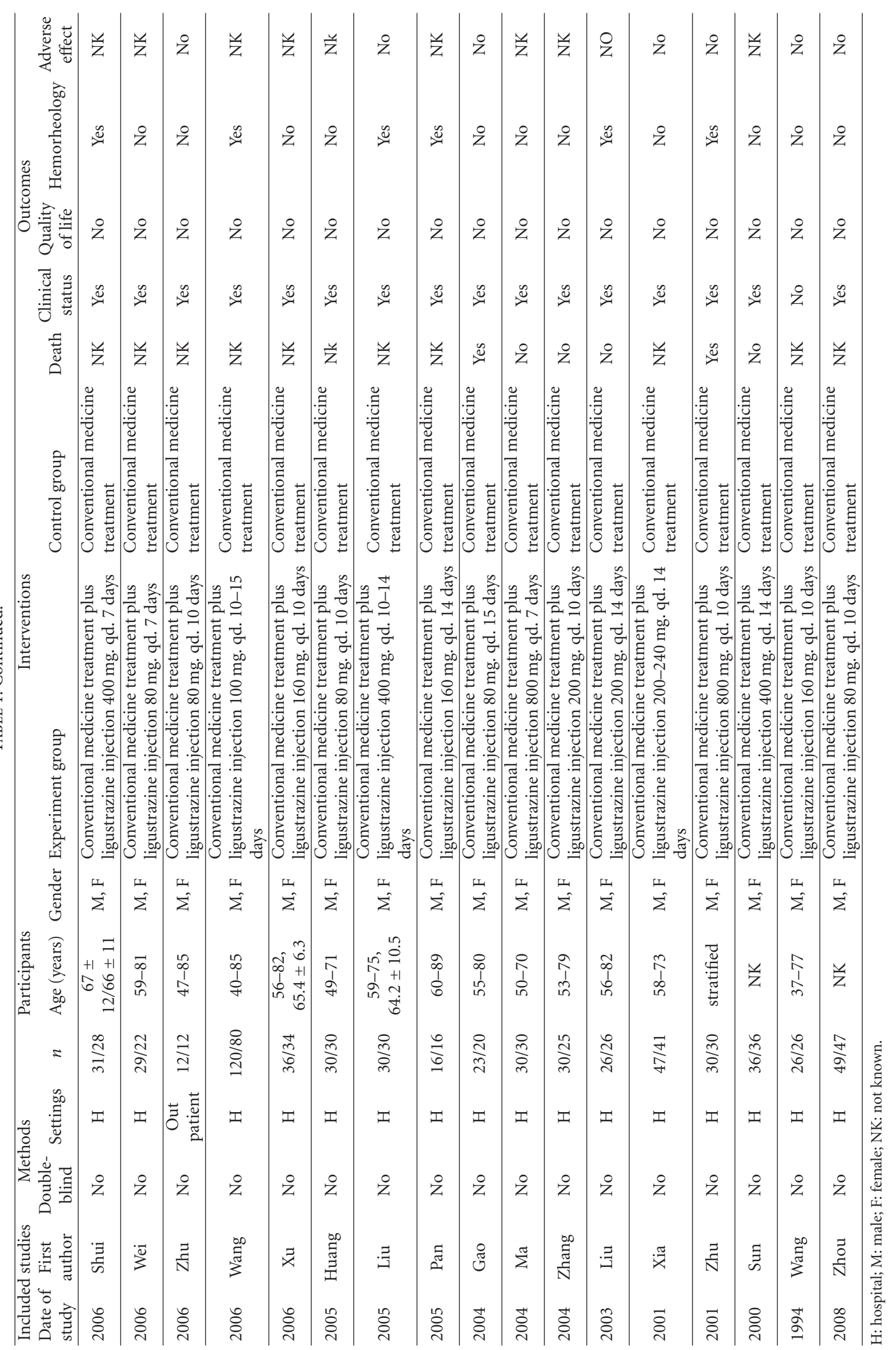


3.1.1. Study of Quality. The methodological quality of most included trials was generally "poor." No trials reported using randomization method, yet only 1 trial mentioned doubleblind [3]; none of the trials was properly randomized, double blinded, placebo controlled. None of the trials had grade a level of adequate concealment of randomization. The remaining 33 possible RCTs did not describe the way of randomization. None of the trials blinded the assessment of outcome. None of the trials reported the number of patients that were lost to followup and whether they had used intention-to-treat analysis.

3.2. Publication Bias. Although we conducted comprehensive searches and tried to avoid bias, since all trials were published in Chinese, we could not exclude potential publication bias.

\subsection{Primary Outcomes}

3.3.1. New York Heart Association Classification of Clinical Status (NYHA Class Improved <1 Class). 32 trials (2319 patients) concluded an improvement in heart failure [334]. The entire heart function classification system used the NYHA functional classification method. A meta-analysis of data on change of NYHA functional classification showed that ligustrazine injection plus conventional treatment had more benefit compared with conventional treatment alone. The summary odds ratio (OR) of NYHA class improved $<$ I class, or worsening of heart failure was 0.22 (OR, 0.22; 95\% CI, 0.17 to 0.28 ) (Figure 2).

\section{Adverse Events.}

The duration of treatment in all included studies was short (7 to 15 Days). Outcomes were measured at the End of treatment. No obvious adverse events occurred in 21 trials, and no adverse events were reported in 11 trials. One trial reported adverse events [17], and the adverse events were described as dry mouth and mild drowsiness, but they were not severe. It will recover without special treatment. There were no adverse events in control group. Although we did not find any related information about adverse effects recorded, potential or long-time adverse effects in the included trials could not be excluded.

\subsection{Secondary Outcomes.}

3.4.1. Death. Deaths were reported in only 3 trials $[27,32$, 35]. The total duration of treatment varied from 10 to 15 days. No death cases reported in the remaining trials. There was no statistically significant difference between 2 groups (OR, 0.74; 95\% CI, 0.19 to 2.81) (Figure 3).

3.4.2. Hemodynamics. Two trials provided data for Doppler echocardiography hemodynamic change $[11,22]$. At the end of treatment, one trial showed increased ejection fraction (weighted mean difference (WMD) $0.09,95 \%$ CI 0.07 to
$0.11)[11,22]$ of ligustrazine injection plus conventional medicine treatment group. One trial showed a significant increase in cardiac output (mean of the experimental group and control group was $4.46 \mathrm{~L} / \mathrm{min}$ to $3.07 \mathrm{~L} / \mathrm{min}$ ), increased ejection fraction (mean of the experimental group and control group was $71 \%$ to $56.9 \%$ ) and increased stroke volume (mean of the experimental group and control group is $56.8 \mathrm{~mL}$ to $36.8 \mathrm{~mL}$ ) [11], and there was significant difference between the two groups, but unfortunately the trial did not provide proper statistic analysis (no standard deviation).

Three trials showed a significant decrease mean pulmonary arterial pressure (WMD $-4.77,95 \%$ CI -5.85 to $-3.68)[11,19,36]$ (Figure 4).

\subsubsection{Quality of Life. No trial assessed quality of life.}

\section{Discussion}

In many years, western medicine has made great progress and had become the dominating and leading medical treatment research and development worldwide. However, it has been increasingly recognized that western medicine may sometimes fail to treat any illness, whereas such illness is reportedly improved by the so-called complementary and alternative medicine based on a different theory. Few relevant articles on TCM for CPHD have been published in the English medical journals, and the limited evaluation of TCM beyond China reduces its external validity.

This review of randomized trials shows the current evidence in ligustrazine injection for CPHD. Treatment was applied according to a diagnosis of CPHD without further syndrome differentiation according to traditional Chinese methodology. Study sizes were generally insufficient. All conclusions, therefore, were impropriate. There were no descriptions of allocation concealment and no assurances that blinding was maintained. The type of conventional medicine used and the dosages were often impropriate reported, so it was not certain that all studies used similar medications.

Ligustrazine injection plus conventional medicine treatment showed significantly less outcomes of decreased heart function as with "no change or worse" in comparison with the treatment of conventional medicine alone. With measurement using NYHA of clinical status (OR, 0.22; 95\% CI, 0.17 to 0.28 ), the treatment group had, on average, 22 percent less than those treated with conventional medicine alone. These results were positively encouraging and promising of combining ligustrazine injection with conventional treatment, which might be beneficial to heart function. The positive findings were also observed in mean pulmonary arterial pressure outcome (WMD $-4.77,95 \% \mathrm{CI}-5.85$ to -3.68). In our opinion, such an average difference would be noticeable and clinically meaningful. In addition, patients treated with ligustrazine injection may have a beneficial effect on cardiac output, ejection fraction, and stroke volume, however, there is just one study providing this result. As for other outcomes, such as death, there were just 3 trials (total $n=215$ ), which showed no significant difference in favour 


\begin{tabular}{|c|c|c|c|c|c|c|c|c|c|}
\hline \multirow{2}{*}{$\begin{array}{l}\text { Study or subgroup } \\
\text { Chenyanhua1999 }\end{array}$} & \multicolumn{2}{|c|}{ Experimental } & $\begin{array}{r}\text { Cor } \\
\text { Events } \\
\end{array}$ & $\begin{array}{l}\text { ntrol } \\
\text { Total } \\
\end{array}$ & Weight & $\begin{array}{c}\text { Odds ratio } \\
\mathrm{M}-\mathrm{H} \text {, fixed, } 95 \% \text { CI }\end{array}$ & \multicolumn{2}{|c|}{$\begin{array}{c}\text { Odds ratio } \\
\text { M-H, fixed, 95\% CI }\end{array}$} & \\
\hline & 2 & 72 & 24 & 52 & $9.3 \%$ & $0.03[0.01,0.15]$ & & & \\
\hline Huangmeixing 2005 & 0 & 30 & 6 & 30 & $2.2 \%$ & $0.06[0,1.15]$ & & & \\
\hline Wangwei 1994 & 1 & 26 & 7 & 26 & $2.3 \%$ & $0.11[0.01,0.96]$ & & & \\
\hline Shuiyaoting 2006 & 1 & 31 & 6 & 28 & $2.1 \%$ & $0.12[0.01,1.09]$ & & & \\
\hline Zhoujianhui 2008 & 2 & 49 & 11 & 47 & $3.7 \%$ & $0.14[0.03,0.67]$ & & & \\
\hline Fanshaodong 2007 & 2 & 36 & 10 & 34 & $3.3 \%$ & $0.14[0.03,0.7]$ & & & \\
\hline Liujian 2005 & 2 & 30 & 10 & 30 & $3.2 \%$ & $0.14[0.03,0.72]$ & & & \\
\hline Zhuyingzi 2006 & 2 & 12 & 7 & 12 & $2 \%$ & $0.14[0.02,0.96]$ & & & \\
\hline Haoruifeng 2009 & 2 & 50 & 11 & 50 & $3.6 \%$ & $0.15[0.03,0.71]$ & & & \\
\hline Wangjing 2006 & 8 & 120 & 24 & 80 & $9.3 \%$ & $0.17[0.07,0.39]$ & & & \\
\hline Qiyi 2008 & 2 & 34 & 8 & 30 & $2.8 \%$ & $0.17[0.03,0.89]$ & & & \\
\hline Zhuming 2001 & 2 & 30 & 8 & 30 & $2.6 \%$ & $0.2[0.04,1.02]$ & & & \\
\hline Yeguoxiang 2008 & 2 & 32 & 8 & 32 & $2.6 \%$ & $0.2[0.04,1.03]$ & & & \\
\hline Sunjiangtao 2000 & 3 & 36 & 11 & 36 & $3.5 \%$ & $0.21[0.05,0.82]$ & & & \\
\hline Zhangshunfeng 2004 & 2 & 30 & 6 & 25 & $2.1 \%$ & $0.23[0.04,1.24]$ & & & \\
\hline Xiaweixin 2001 & 5 & 47 & 14 & 41 & $4.6 \%$ & $0.23[0.07,0.71]$ & & & \\
\hline Wuliwen 2009 & 2 & 30 & 7 & 30 & $2.3 \%$ & $0.23[0.04,1.24]$ & & & \\
\hline Chendongyun 2010 & 2 & 46 & 7 & 46 & $2.3 \%$ & $0.25[0.05,1.29]$ & & & \\
\hline Anyufen 2009 & 3 & 40 & 9 & 38 & $2.9 \%$ & $0.26[0.06,1.05]$ & & & \\
\hline Lilanju 2008 & 4 & 31 & 11 & 31 & $3.3 \%$ & $0.27[0.07,0.97]$ & & & \\
\hline Baishurong 2009 & 3 & 35 & 9 & 35 & $2.8 \%$ & $0.27[0.07,1.1]$ & & & \\
\hline Liuqingyi 2008 & 2 & 38 & 6 & 36 & $2 \%$ & $0.28[0.05,1.48]$ & & & \\
\hline Maxin 2004 & 2 & 30 & 6 & 30 & $1.9 \%$ & $0.29[0.05,1.55]$ & & & \\
\hline Panxifeng2005 & 1 & 16 & 3 & 16 & $1 \%$ & $0.29[0.03,3.13]$ & & & \\
\hline Xuping 2006 & 4 & 36 & 10 & 34 & $3.2 \%$ & $0.3[0.08,1.07]$ & & & \\
\hline Wuxia 2009 & 3 & 40 & 8 & 40 & $2.6 \%$ & $0.32[0.08,1.33]$ & & & \\
\hline Weijun 2006 & 4 & 29 & 7 & 22 & $2.4 \%$ & $0.34[0.09,1.37]$ & & & \\
\hline Xielongguo 2008 & 8 & 56 & 16 & 49 & $5 \%$ & $0.34[0.13,0.9]$ & & & \\
\hline Sunlili 2007 & 5 & 40 & 10 & 40 & $3 \%$ & $0.43[0.13,1.39]$ & & & \\
\hline Yuanrongzheng 2010 & 10 & 31 & 16 & 31 & $3.7 \%$ & $0.45[0.16,1.25]$ & & & \\
\hline Liuzhong 2003 & 2 & 26 & 4 & 26 & $1.3 \%$ & $0.46[0.08,2.75]$ & & & \\
\hline Gaoyanqiu 2004 & 2 & 23 & 3 & 20 & $1 \%$ & $0.54[0.08,3.61]$ & & & \\
\hline Total $(95 \%$ CI $)$ & & 1212 & & 107 & $100 \%$ & $0.22[0.17,0.28]$ & 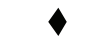 & & \\
\hline Total events & 95 & & 303 & & & & & & \\
\hline Heterogeneity: $\mathrm{Chi}^{2}=$ & $=16.1$ &, $\mathrm{df}=$ & $1(P=$ & $0.99)$ & $I^{2}=0 \%$ & 0.0 & 1 & $\begin{array}{c}1 \\
10\end{array}$ & 100 \\
\hline
\end{tabular}

FIgURE 2: Comparison of ligustrazine hydrochloride injection + conventional treatment versus conventional treatment. Lack of improvement in heart failure (NYHA class improved < I class or worsening of heart failure). M-H: Mantel-Haenszel formula.

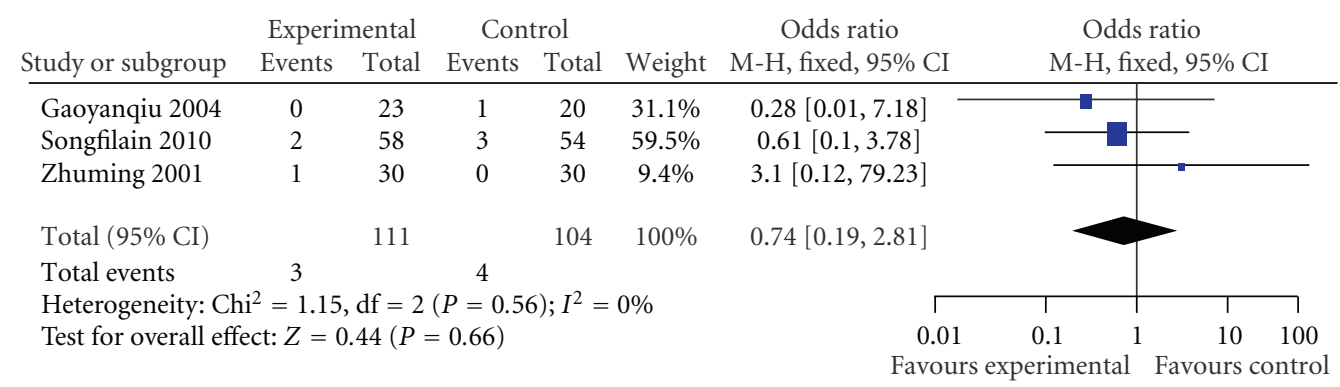

FIGURE 3: Comparison of death rate in ligustrazine hydrochloride injection + conventional treatment versus conventional treatment.

of the ligustrazine injection plus conventional combination treatment (OR, $0.74 ; 95 \% \mathrm{CI}, 0.19$ to 2.81 ).

Adverse effects were reported by Shaodong and Xuefen (2007) [17] as only dry mouth and mild drowsiness, and the adverse events were not severe. They spontaneously recovered without special treatment. No obvious adverse events occurred in 21 trials. 11 trials did not report adverse effects and these were not significantly different between the two treatments. However, the concrete conclusion regarding safety cannot be determined from this review due to the limited evidence provided by the eligible trials. In order to proper assess the safety of ligustrazine injection, large-scale clinical trials with long-term followup are required.

The application of traditional Chinese herbal medicine is fundamentally prescribed with syndrome differentiation. Failure to apply syndrome differentiation may result in 


\begin{tabular}{|c|c|c|c|c|c|c|c|c|c|c|c|}
\hline \multirow[b]{2}{*}{ Study or subgroup } & \multicolumn{3}{|c|}{ Experimental } & \multicolumn{2}{|c|}{ Control } & \multirow[b]{2}{*}{ Total } & \multirow[b]{2}{*}{ Weight } & \multirow{2}{*}{$\begin{array}{l}\text { Mean difference } \\
\text { IV, fixed, } 95 \% \text { CI }\end{array}$} & \multirow{2}{*}{\multicolumn{2}{|c|}{$\begin{array}{l}\text { Mean difference } \\
\text { IV, fixed, 95\% CI }\end{array}$}} & \\
\hline & Mean & SD & Total & Mean & $\mathrm{SD}$ & & & & & & \\
\hline Majianguo 2009 & 28.97 & 3.41 & 36 & 32.89 & 4.32 & 36 & $36.4 \%$ & $-3.92[-5.72,-2.12]$ & 5 & & \\
\hline Shuiyaoting 2006 & 20.6 & 1.9 & 31 & 25.7 & 4.3 & 28 & $39.4 \%$ & $-5.1[-6.83,-3.37]$ & 마 & & \\
\hline Zhoujianhui 2008 & 32.1 & 6 & 49 & 37.6 & 5 & 47 & $24.2 \%$ & $-5.5[-7.71,-3.29]$ & - & & \\
\hline Total $(95 \%$ CI $)$ & & & 116 & & & 111 & $100 \%$ & $-4.77[-5.85,-3.68]$ & 1 & & \\
\hline \multicolumn{8}{|c|}{ Heterogeneity: $\mathrm{Chi}^{2}=1.42, \mathrm{df}=2(P=0.49) ; I^{2}=0 \%$} & $\begin{array}{r}{ }_{-100} \\
\end{array}$ & -50 & $\begin{array}{ll}1 \\
50\end{array}$ & 100 \\
\hline
\end{tabular}

FIGURE 4: Comparison of mean pulmonary arterial pressure in ligustrazine hydrochloride injection + conventional treatment versus conventional treatment. SD: standard deviation.

treatments being ineffective or even harmful. Despite this, there is some evidence that these Chinese herbal medicines, combined with conventional medicine and given in a way that is not in keeping with their normal use within traditional Chinese medicine, may be beneficial for people with CPHD across a range of outcomes. If these medicines are used properly the positive effects could be enhanced.

The improvement of heart failure was noticed in the survey; however, the effects may not be true due to improper study design or method used, such as lack of randomization or allocation concealment. Publication bias could also be a factor. we tried to take all measures to contact authors to get further information either by telephone, letter, or email. Unfortunately, we got no replies, and we are not sure, the trials were conducted as true RCT. Subsequently, no clear conclusion could be made from these trials.

\section{Conclusions}

In conclusion, because of the unclear methodological quality of these identified trials, a definite conclusion on efficacy and adverse events associated with ligustrazine injection cannot be drawn from this review. We found no evidence of a beneficial effect on the primary measure of efficacy to support the routine use of ligustrazine injection for CPHD. However the drug appeared to have no major adverse effects, and if its positive effects were confirmed by comprehensive clinical trials, it would lead to many promising treatments for CPHD and could benefit all patients all over the world. Therefore, further thorough investigation, large-scale, proper study design, randomized trials of ligustrazine injection for CPHD will be required to justify the effects reported here. Future trials should overcome the limitations of the trials presented in this review; particularly, they should assure adequate concealment of allocation and blinding of outcome assessors and use functional outcome as the primary outcome measured at long-term followup. Reports of the trials should conform to the recommendations of the CONSORT statement. If reliable RCT results confirmed ligustrazine injection's positive effects for treatment of CPHD, it would be blessing news for CPHD patients worldwide.

\section{Acknowledgments}

This paper is supported by the National Program on Key Basic Research Project (973 Program) (Grant no. 2006CB504605) and the Program for New Century Excellent Talents in the University of HeNan Province (Grant no. 2006HANCET-05). Li Jian-sheng and W. Hai-feng are contributing equally to this work.

\section{References}

[1] E. Weitzenblum, “Chronic cor pulmonale," Heart, vol. 89, no. 2, pp. 225-230, 2003.

[2] A. R. Jadad, R. A. Moore, D. Carroll et al., "Assessing the quality of reports of randomized clinical trials: Is blinding necessary?" Controlled Clinical Trials, vol. 17, no. 1, pp. 1-12, 1996.

[3] C. Yanhua, "Clinical analysis of high-dose ligustrazine in treatment of pulmonary heart disease: an observation of 72 cases," Henan Medical Information, vol. 7, no. 2, p. 51, 1999.

[4] C. Dongyun, "Clinical analysis of ligustrazine injection in adjuvant treatment of pulmonary heart disease: an observation of 46 cases," China Modern Doctor, vol. 48, no. 9, pp. 100108, 2010.

[5] Y. Rongzheng, "Clinical observation of ligustrazine hydrochloride injection in treating pulmonary heart disease," China Modern Medicine, vol. 17, no. 27, pp. 72-73, 2010.

[6] B. Shurong and W. Yuan, "Influence of ligustrazine injection on hemorrheology in patients with acute exacerbation of pulmonary heart disease," Shandong Medical Journal, vol. 49, no. 41 , pp. 55-56, 2009.

[7] A. Yufen and Z. Xinli, "Treating 40 cases of acute exacerbation of chronic pulmonary heart disease with ligustrazine injection," Chinese Journal of the Practical Chinese with Modern Medicine, vol. 22, no. 19, pp. 1520-1521, 2009.

[8] H. Ruifeng, "Effect of ligustrazine in treatment of pulmonary heart disease with acute exacerbation," Journal of Shanxi Medical College for Continuing Education, vol. 19, no. 4, p. 35, 2009.

[9] W. Liwen, "Clinical observation of ligustrazine Injection in treatment of chronic pulmonary heart disease heart failure: an observation of 30 cases," Journal of Xinjiang Medical University, vol. 7, p. 929, 2009.

[10] W. Xia, "The nursing of using ligustrazine to cure chronic pulmonary heart disease heart failure," Modern Journal of Integrated Traditional Chinese and Western Medicine, vol. 18, no. 6 , p. 679, 2009. 
[11] M. Jianguo, "Influence of ligustrazine injection on heart function in patients with chronic pulmonary heart disease," China Practical Medicine, vol. 4, no. 8, p. 154, 2009.

[12] L. Lanju, W. Yiping, L. Ruifang et al., "Clinical observation of ligustrazine injection in treatment of chronic pulmonary heart disease with acute exacerbation: an observation of 31 cases," Journal of Hebei Traditional Chinese Medicine and Pharmacology, vol. 23, no. 2, pp. 10-11, 2008.

[13] L. Qingyi and Z. Huiying, "Influence of ligustrazine injection on hemorrheology in patients with acute exacerbation of pulmonary heart disease ," Clinical Medicine, vol. 28, no. 6, pp. 6-7, 2008.

[14] Q. yi, "Clinical observation of Ligustrazine injection in treatment of pulmonary heart disease with hyperviscosity syndrome," Journal of Mudanjiang Medical College, vol. 29, no. 2, pp. 35-36, 2008.

[15] Y. Guoxiang, "Effect of ligustrazine in treatment of pulmonary heart disease with acute exacerbation," The Medical Journal of Industrial Enterprise, vol. 21, no. 6, pp. 45-46, 2008.

[16] X. Longguo, "Clinical observation of ligustrazine injection in treatment of pulmonary heart disease heart failure," Journal of Qiqihar Medical College, vol. 29, no. 17, pp. 2095-2096, 2008.

[17] F. Shaodong and W. Xuefen, "Influence of ligustrazine injection on hemorrheology in patients of chronic pulmonary heart disease with acute exacerbation," Zhejiang Clinical Medical Journal, vol. 9, no. 12, pp. 1670-1671, 2007.

[18] S. Lili, S. Dan, and W. Junbo, "Clinical observation of ligustrazine injection in treatment of pulmonary heart disease with acute exacerbation," Chinese Journal of Postgraduates of Medicine, vol. 30, no. z1, pp. 163-164, 2007.

[19] S. Yaoting and Z. Jianguo, "Clinical observation of ligustrazine injection in treatment of chronic pulmonary heart disease with acute exacerbation," Journal of Chinese Modern Traditional Chinese Medicine, vol. 2, no. 8, pp. 709-710, 2006.

[20] W. Jun, "Clinical analysis of ligustrazine injection in treatment of chronic pulmonary heart disease heart failure," Modern Journal of Integrated Traditional Chinese and Western Medicine, vol. 15, no. 11, pp. 1428-1429, 2006.

[21] Z. Yingzi, "Clinical analysis of ligustrazine hydrochloride injection in treating chronic pulmonary heart disease heart failure: an observation of 24 cases," Chinese Community Doctors, vol. 8, no. 3, p. 37, 2006.

[22] W. Jing, L. I. Zhongqing, and S. Ning, "Effect of ligustrazine injection in treatment of chronic pulmonary heart disease," Chinese Journal of Integrative Medicine on Cardio/Cerebrovascular Disease, vol. 4, no. 12, pp. 1042-1043, 2006.

[23] X. Ping and L. Jianping, "Clinical analysis of ligustrazine injection in treating chronic pulmonary heart disease with acute exacerbation: an observation of 36 cases," Jiangxi Journal of Traditional Chinese Medicine, vol. 37, no. 12, pp. 25-26, 2006.

[24] H. Meixing, C. Xueyuan, T. Yuping et al., "Influence of ligustrazine injection on lung function and vaso-activesubstance in patients of chronic pulmonary heart disease with acute exacerbation," The Journal of Practical Medicine, vol. 21, no. 3, pp. 1479-1480, 2005.

[25] L. Jian and Z. Lihong, "Clinical observation of ligustrazine injection in treatment of pulmonary heart disease with acute exacerbation," Practical Clinical Medicine, vol. 6, no. 9, pp. 5354, 2005.

[26] P. Xifeng, C. Ping, K. Yating et al., "Influence of ligustrazine injection on hemorrheology in elderly patients with pul- monary heart disease," Chinese Journal of Microcirculation, vol. 15 , no. 4, pp. 57-58, 2005.

[27] Gaoyanqiu, M. Wei, and Y. Ning, "Clinical observation of ligustrazine Injection in treatment of chronic pulmonary heart disease," Clinical Medicine, vol. 24, no. 9, pp. 24-25, 2004.

[28] M. Xin, "Clinical analysis of ligustrazine injection in treating chronic pulmonary heart disease heart failure: an observation of 30 cases," Journal of Handan Medical College, vol. 17, no. 1, pp. 20-21, 2004.

[29] Z. Shunfeng, "Clinical analysis of ligustrazine injection in treating pulmonary heart disease heart failure: an observation of 30 cases," Journal of Medical Forum, vol. 25, no. 21, p. 43, 2004.

[30] L. Zhong, "Influence of ligustrazine injection on hemorrheology in patients with chronic pulmonary heart disease," China Journal of Modern Medicine, vol. 13, no. 10, pp. 84-85, 2003.

[31] X. Weixin and Z. Hua, "Clinical analysis of ligustrazine injection in treating pulmonary heart disease heart failure: an observation of 47 cases," Clinical analysis of ligustrazine injection in treating pulmonary heart disease heart failure: an observation of 47 cases, vol. 10, no. 7, p. 635, 2001.

[32] Z. Ming, "Clinical analysis of ligustrazine injection in treating chronic pulmonary heart disease: an observation of 30 cases," Journal of Bengbu Medical College, vol. 26, no. 3, pp. 247-248, 2001.

[33] S. Jiangtao, "Clinical analysis of ligustrazine injection in adjuvant treatment of pulmonary heart disease: an observation of 34 cases," Modern Medicine, vol. 13, no. 2, pp. 8-9, 2000.

[34] W. Wei and Z. Yanping, "Clinical observation of ligustrazine injection in treatment of pulmonary heart disease with acute exacerbation," Jiangxi Journal of Traditional Chinese Medicine, vol. 25, no. 2, pp. 26-27, 1994.

[35] S. Fulian and Q. Yuan, "Therapeutic effect of LigustrazineHydrochloride Injection on chronic pulmonary heart disease combined with right heart failure," China Medical Herald, vol. 7, no. 12, pp. 83-84, 2010.

[36] Z. Jianhui, J. Zehuan, Liuyang et al., "Influence of ligustrazine injection on pulmonary arterial pressure in patients with chronic pulmonary heart disease," Journal of Chinese Modern Medicine, vol. 5, no. 1, pp. 41-42, 2008. 


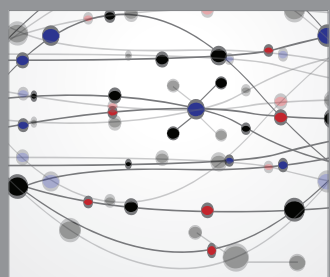

The Scientific World Journal
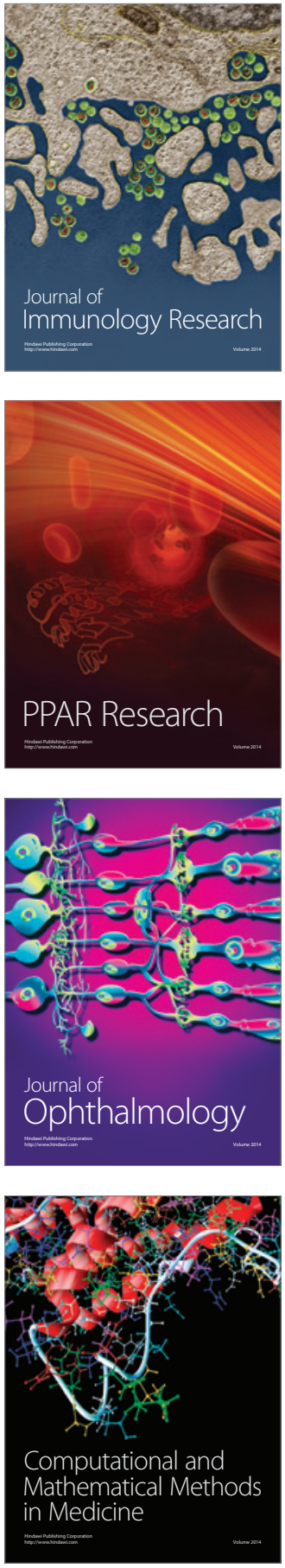

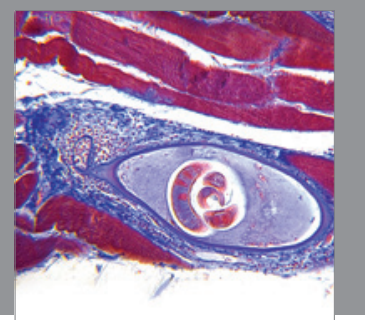

Gastroenterology

Research and Practice
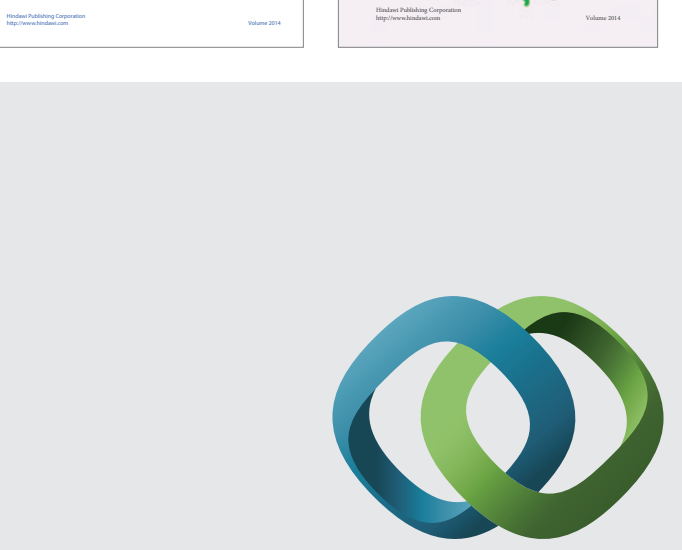

\section{Hindawi}

Submit your manuscripts at

http://www.hindawi.com
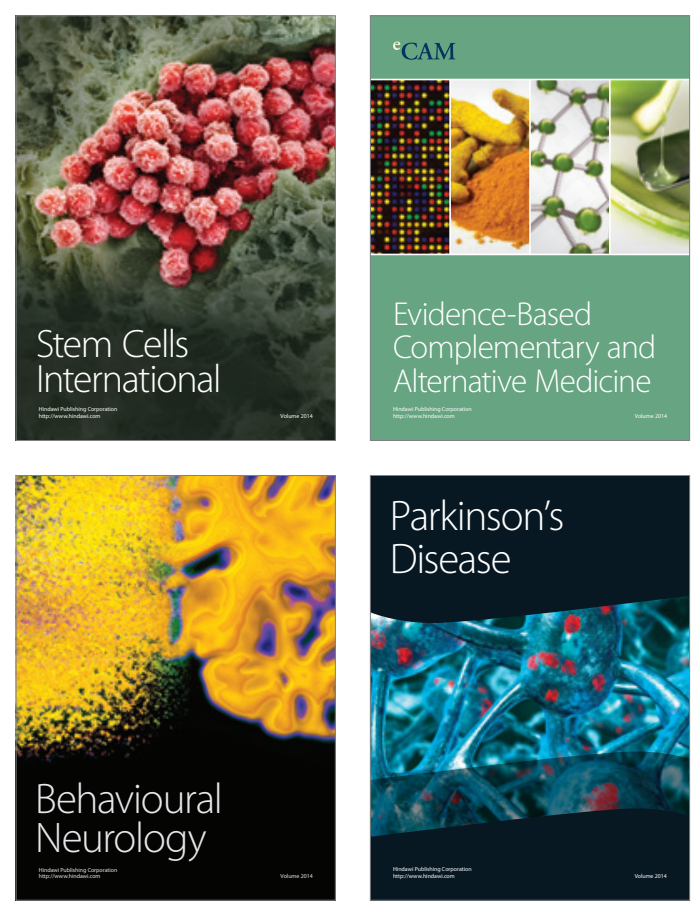

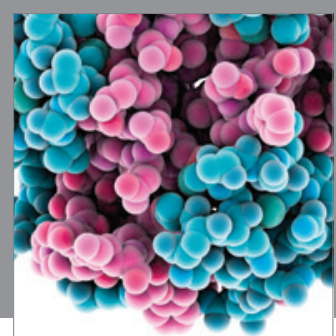

Journal of
Diabetes Research

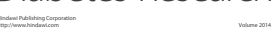

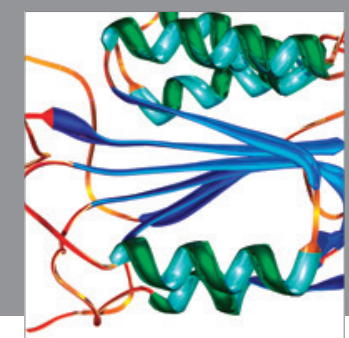

Disease Markers
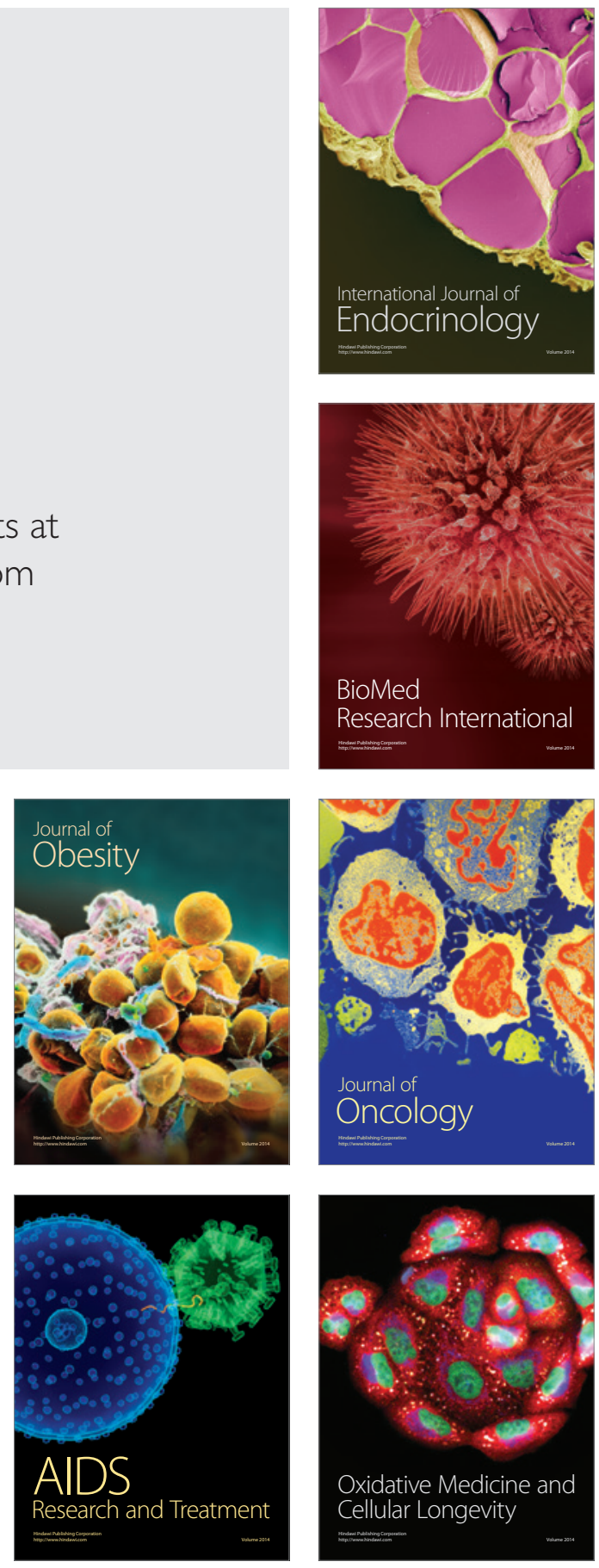